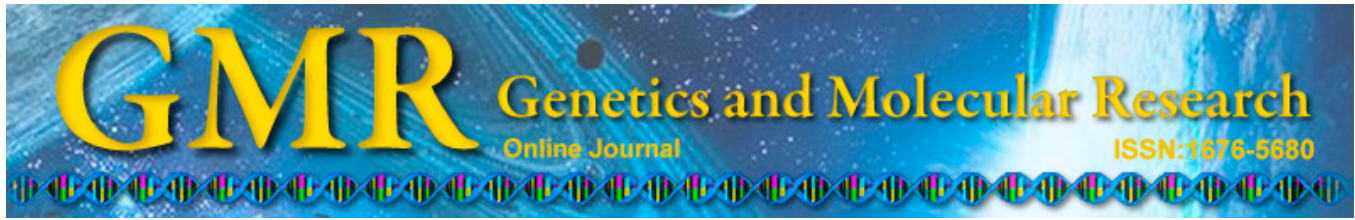

\title{
Study on the correlation between the expression of Ki67 and FasL and prognosis of cervical carcinoma
}

\author{
S.N. Liang ${ }^{1}$, Y.J. Huang ${ }^{2}$, L.L. Liu ${ }^{3}$ and X. Liu ${ }^{4}$ \\ ${ }^{1}$ The First Affiliated Hospital of China Medical University, Shenyang, \\ Liaoning, China \\ ${ }^{2}$ Jiangxi Maternal and Child Health Hospital, Jiangxi, China \\ ${ }^{3}$ China Medical University, Shenyang, Liaoning, China \\ ${ }^{4}$ No. 202 Hospital of PLA, Shenyang, Liaoning, China \\ Corresponding author: S.N. Liang \\ E-mail: songnianliang@126.com
}

Genet. Mol. Res. 14 (3): 8634-8639 (2015)

Received September 30, 2014

Accepted March 18, 2015

Published July 31, 2015

DOI http://dx.doi.org/10.4238/2015.July.31.11

\begin{abstract}
This study aimed to investigate the correlation between the expression of Ki67 and FasL and prognosis of cervical carcinoma and to explore the biological processes and signal pathways related to cervical carcinoma. Cervical carcinoma tissue specimens from 200 patients and normal tissue specimens adjacent to lesions from 30 cases were collected in this study. Ki67 and FasL proteins in these specimens were detected by immunohistochemical methods. A series of statistical methods were carried out to investigate the correlation between the expression of Ki67 and FasL and prognosis of cervical carcinoma. The expression of Ki67 and FasL in cervical carcinoma tissues was significantly higher than that in normal cervical tissue. The positive rate of Ki67 and FasL increased with the increase in the degree of cervical lesions. There was a positive correlation between the expression of Ki67 and FasL in cervical lesions. The expression
\end{abstract}


of Ki67 and FasL affected the five-year survival rate of postoperative patients. Ki67 and FasL were independent factors for the prognosis of patients with cervical cancer. The expression of Ki67 and FasL is closely related to the occurrence and development of cervical carcinoma. There is a positive correlation between Ki67 and FasL, and they may be biomarkers of cervical cancer.

Key words: Ki67; FasL; Cervical carcinoma; Prognosis

\section{INTRODUCTION}

Cervical cancer is the second most common malignant tumor affecting female health. Each year in the world, about 500,000 women are diagnosed with this disease and 200,000 die of this disease (Schoell et al., 1999; Chan et al., 2002). Most cases of cervical cancer are due to infection by human papillomavirus caused by sexual contact. It has a long latency and has no obvious symptoms in the early stage. It causes pelvic pain and vaginal bleeding in the late stage. At present, there are no effective drugs for the treatment of cervical cancer. Thus, it is difficult to cure and recurrence rate is high.

The Ki67 gene is the most important driving gene in cervical cancer chromosome $10 \mathrm{q} 25$ region, where its overexpression is often associated with the over amplification of the 10q25 region (Combita et al., 2002). Previous studies indicated that Ki67 was an important potential cancer gene and that it was overexpressed in luminal B type cervical cancer. It affects the progression of cervical cancer by inhibiting the transcription of the target gene (Hagensee, 2000; Reinstein et al., 2000). But so far, the relationship between the expression of Ki67 and clinical pathological data of cervical carcinoma and the association of Ki67 with the prognosis of cervical cancer are not clear.

We studied cervical tumor tissue from 200 cases and normal tissue adjacent to cervical tumor from 30 cases, using immunohistochemical methods, and explored the relationship of Ki67 expression to clinical pathological data and prognosis of cervical carcinoma.

\section{MATERIAL AND METHODS}

\section{Subjects}

A total of 200 tissue specimens were obtained from patients with cervical cancer (36-83 years old, average of 55.9 years) after tumor resection at the First Affiliated Hospital of China Medical University. The types of cervical cancer included 50 cases of luminal A, 90 cases of luminal B, 30 cases of Her2 overexpression, and 30 cases of basal-like. Thirty specimens of normal tissue adjacent to tumor were selected randomly from the above specimens. Exclusion criteria were: patients dead within one month after the operation, metastasis to other organs, history of other tumors, and preoperative chemotherapy and biological therapy. Cervical cancer was staged according to the TNM classification of malignant tumor (Daemen et al., 2002), and molecular typing was according to Natale et al. (2000).

All subjects signed an informed consent form. This study was approved by the Ethics Committee of the China Medical University. 


\section{Immunohistochemical staining}

Pathological surgical tumor specimens fixed in $10 \%$ formaldehyde were taken out and washed, and they were then paraffin-embedded with automatic embedding machine. The embedded specimens were sliced at a thickness of $3 \mu \mathrm{m}$. Following deparaffinization, dehydration, and antigen retrieval, the sections were blocked with $5 \% \mathrm{BSA}$ and incubated at $37^{\circ} \mathrm{C}$ for $20 \mathrm{~min}$, and they were then incubated with 1:50 diluted first antibodies at $4^{\circ} \mathrm{C}$ overnight. Afterwards, they were washed with PBS and incubated at $37^{\circ} \mathrm{C}$ in a water bath for $2 \mathrm{~h}$ after adding the 2nd antibody, followed by washing with PBS. After treating with DAB solution, the slides were flushed completely, counterstained with hematoxylin, washed with water, dried, coverslipped, and observed under a light microscope.

Immunohistochemical staining was scored by a pathologist and an author separately so as to minimize subjective factors; the scores were compared to get the final comprehensive results. The final score of staining results $=$ score of the staining intensity (A) $\mathrm{x}$ score of positive cell percent (B). Score A was as follows: $0=$ no signal, $1=$ weak, $2=$ moderate, and $3=$ marked. Score B was as follows: $1-25 \%=1,26-50 \%=2,51-75 \%=3$, and $76-100 \%=4$. Each section was examined at 400X magnification, evaluating 100 cells in each of 5 continuous high power fields, and then taking the mean value. A final score $=0$ was considered negative $(-) ; \leq 4$, low expression $(+) ; 5-8$, moderate expression $(++) ; \geq 9$, high expression $(+++)$.

\section{Data analysis}

Statistical analysis was performed using Statistical Package for the Social Science (SPSS, version 17.0). The Pearson chi-square or the Fisher exact test was used to analyze the clinical pathological data. The survival curve was drawn using the Kaplan-Meier statistical method. The prognosis data were analyzed with single and multifactor Cox regression analysis. $\mathrm{P}<0.05$ was considered to be significant.

\section{RESULTS}

\section{Immunohistochemical detection results}

Ki67 protein was mainly localized in the intranuclear region of epithelial cells, with a few cases of expression in the cytoplasm, but there was no expression in mesenchymal cells (Figure 1). The expression of Ki67 and FasL was higher in luminal B type of cervical cancer than in the other types of cervical cancer and adjacent normal tissues $(\mathrm{P}<0.05$, Table 1$)$.

\section{Correlation between expression of Ki67 and clinical pathological data of luminal B type of cervical cancer}

The results showed that there was a correlation between the expression of Ki67 and tumor size, lymph node metastasis and TNM staging of luminal B type of cervical cancer $(\mathrm{P}<$ 0.05). There was no significant relationship between the expression of Ki67 and patient's age, menopausal status or SBR classification of cervical cancer $(P>0.05)$. At the same time, we also found that there was a positive correlation between the expression of Ki67 and FasL $(\mathrm{P}<0.05)$. 


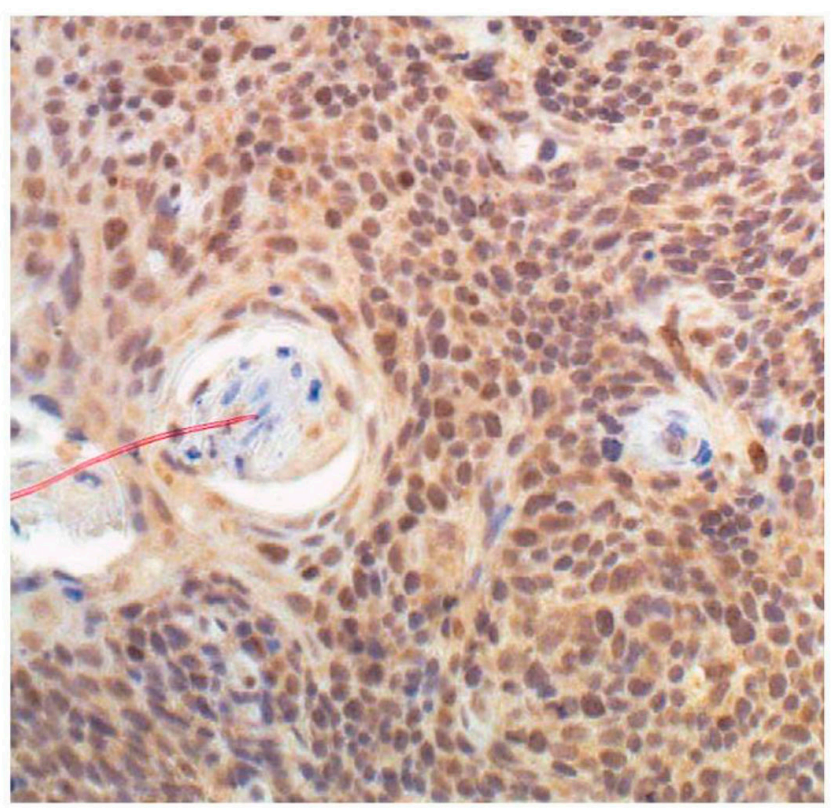

Figure 1. Immunohistochemical detection results of Ki67. Ki67 protein was mainly localized in the intranuclear of epithelial cells, there was also a few expressions in the cytoplasm, but there was no expression in mesenchymal cells.

Table 1. Expression of Ki67 and FasL in different type of cervical cancer and pericarcinoma normal tissues.

\begin{tabular}{lcccc}
\hline Type & Ki67+ & Ki67- & FasL+ & FasL- \\
\hline Luminal B & 14 & 16 & 15 & 15 \\
Luminal A & 3 & $27^{*}$ & 2 & $28^{*}$ \\
Her2 overexpression & 1 & $29^{*}$ & 2 & $28^{*}$ \\
Basal-like & 1 & $29^{*}$ & 1 & $29^{*}$ \\
Pericarcinoma normal tissues & 2 & $28^{*}$ & 1 & $29^{*}$ \\
\hline
\end{tabular}

*Compared with luminal $\mathrm{B}, \mathrm{P}<0.05$.

\section{Correlation between the expression of Ki67 and FasL and cancer-free survival and overall survival time of luminal B type of cervical cancer}

Kaplan-Meier survival analysis showed that the cancer-free and overall survival time in the group with luminal B type of cervical cancer and low Ki67 expression was longer than that of the high Ki67 expression group (Figure 2A and B; P $<0.05$ ). However, the cancer-free and overall survival time of the group with luminal B type cervical cancer with low FasL expression was shorter than that of the high FasL expression group (Figure $2 \mathrm{C}$ and $\mathrm{D} ; \mathrm{P}<0.05)$.

Single and multifactor Cox regression analysis showed that there was a correlation between cancer-free and overall survival time and the expression of Ki67 and FasL, tumor size, lymph node metastasis and TNM staging of luminal B type of cervical cancer $(\mathrm{P}<0.05)$, which suggested that the expression of Ki67 and FasL represented independent prognostic indicators of luminal B type of cervical cancer. 

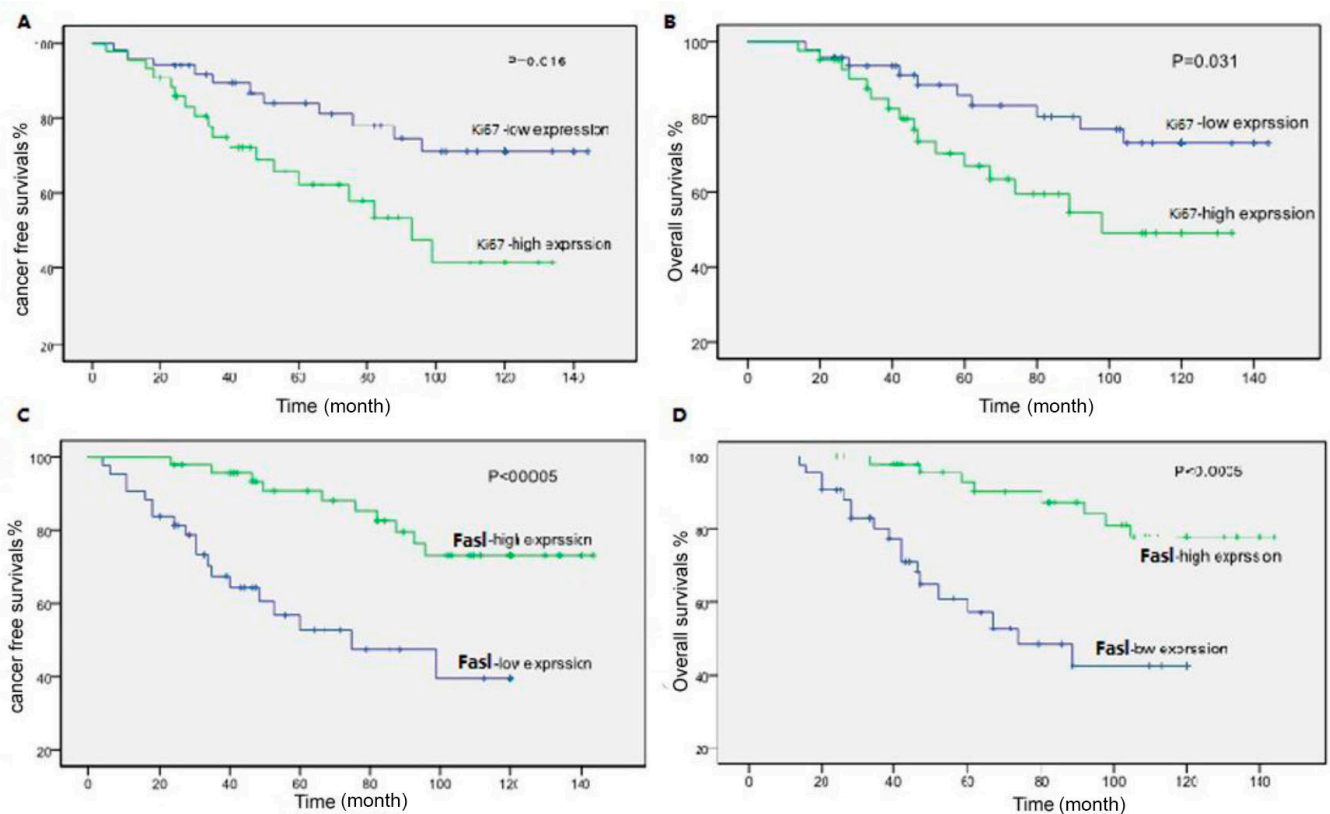

Figure 2. Kaplan-Meier survival analysis. A. Cancer-free survival time in luminal B type of cervical cancer with low-expression of Ki67 group was longer than that of high-expression of Ki67 group $(\mathrm{P}<0.05)$. B. Overall survival time in luminal B type of cervical cancer with low-expression of Ki67 group was longer than that of high-expression of Ki67 group $(\mathrm{P}<0.05)$. C. Cancer free survival time in luminal B type of cervical cancer with low-expression of FasL group was shorter than that of high-expression of FasL group $(\mathrm{P}<0.05)$. D. Overall survival time in luminal $\mathrm{B}$ type of cervical cancer with low-expression of FasL group was shorter than that of high-expression of FasL group $(\mathrm{P}<0.05)$.

\section{DISCUSSION}

Cervical cancer seriously threatens world women's health, so there is an urgent need to study its underlying mechanisms. Cervical cancer leads to a large number of patients dead every year (Villa et al., 2000). The incidence of cervical cancer in different countries and regions showed significant differences (Boyle et al., 2000; von Knebel Doeberitz, 2001). Recent studies showed that immune cells, cytokines and other immune-related regulators play an important role in the development of cervical cancer, including cancer initiation, development, occurrence and metastasis stages (Birnbaum et al., 2003). Exploring the molecular pathogenesis of cervical carcinoma and finding the related target treatment molecules are key approaches for clinical cure and eradication of cervical cancer.

A preliminary study was carried out on the relationship of Ki67 and FasL and cervical cancer. The results suggested that Ki67 can induce the cathepsin activity of tumor-associated macrophages, and promote the ability of proliferation and invasion of tumor (Pereira-Castro et al., 2013). Another study found that Ki67 was an important regulator, which played an important role in promoting tumor growth, angiogenesis and invasion (Nakamura et al., 2008). FasL plays an important role in the synergistic effect of tumor immunity (Slorach et al., 2011). A previous study found that various natural killer cells prompted loss of FasL in deprived tumor cells, which suggested that it played a role in inhibiting tumorigenesis (Sobin et al., 2009). 
In this study, we found that the expression of Ki67 and FasL was higher in luminal B type of cervical cancer than in other types and adjacent normal tissues. Also, there was a correlation between the expression of Ki67 and tumor size, lymph node metastasis and TNM staging of luminal B type of cervical cancer. There was no significant relationship between the expression of Ki67 and patient's age, menopausal status or SBR classification of cervical cancer. At the same time, we also found that there was a positive correlation between the expression of Ki67 and FasL. There was a correlation between cancer-free and overall survival time and the expression of Ki67 and FasL, which suggested that the expression of Ki67 and FasL was an independent prognostic indicator of luminal B type of cervical cancer. Therefore, further study of the expression of Ki67 and FasL and their mechanism of action may have potential value in monitoring the process of cervical cancer and determining metastasis and prognosis. The findings of such study could help in the development of biological agents to reverse tumor immune escape, which is expected to open new areas of cancer therapy.

\section{Conflicts of interest}

The authors declare no conflict of interest.

\section{REFERENCES}

Birnbaum D, Adélaïde J, Popovici C, Charafe-Jauffret E, et al. (2003). Chromosome arm 8p and cancer: a fragile hypothesis. Lancet Oncol. 4: 639-642.

Boyle P, Maisonneuve P and Autier P (2000). Update on cancer control in women. Int J. Gynaecol. Obstet. 70: 263-303.

Chan PK, Chang AR, Cheung JL, Chan DP, et al. (2002). Determinants of cervical human papillomavirus infection: differences between high- and low-oncogenic risk types. J. Infect. Dis. 185: 28-35.

Combita AL, Bravo MM, Touzé A, Orozco O, et al. (2002). Serologic response to human oncogenic papillomavirus types 16, 18, 31, 33, 39, 58 and 59 virus-like particles in Colombian women with invasive cervical cancer. Int. J. Cancer. 97: 796-803.

Daemen T, Regts J, Holtrop M and Wilschut J (2002). Immunization strategy against cervical cancer involving an alphavirus vector expressing high levels of a stable fusion protein of human papillomavirus 16 E6 and E7. Gene Ther. 9: 85-94.

Hagensee ME (2000). Infection with Human Papillomavirus: Update on Epidemiology, Diagnosis, and Treatment. Curr. Infect. Dis. Rep. 2: 18-24.

Nakamura M, Choe SK, Runko AP, Gardner PD and Sagerström CG (2008). Nlz1/Znf703 acts as a repressor of transcription. BMC Dev. Biol. 8: 108.

Natale C, Giannini T, Lucchese A and Kanduc D (2000). Computer-assisted analysis of molecular mimicry between human papillomavirus 16 E7 oncoprotein and human protein sequences. Immunol. Cell Biol. 78: 580-585.

Pereira-Castro I, Costa AM, Oliveira MJ, Barbosa I, et al. (2013). Characterization of Human NLZ1/KI67 Identifies Conserved Domains Essential for Proper Subcellular Localization and Transcriptional Repression. J. Cell Biochem. 114: 120-133.

Reinstein E, Scheffner M, Oren M, Ciechanover A, et al. (2000). Degradation of the E7 human papillomavirus oncoprotein by the ubiquitin-proteasome system: targeting via ubiquitination of the N-terminal residue. Oncogene 19: 5944-5950.

Schoell WM, Janicek MF and Mirhashemi R (1999). Epidemiology and biology of cervical cancer. Semin. Surg. Oncol. 16: 203-211.

Slorach EM, Chou J and Werb Z (2011). Zeppo1 is a novel metastasis promoter that represses E-cadherin expression and regulates p120-catenin isoform expression and localization. Genes Dev. 25: 471-484.

Sobin LH, Gospodarowicz MK, Wittekind C, editors (2009). TNM classification of malignant tumours. 7th edn. West Sussex: Wiley.

Villa LL, Sichero L, Rahal P, Caballero O, et al. (2000). Molecular variants of human papillomavirus types 16 and 18 preferentially associated with cervical neoplasia. J. Gen. Virol. 81: 2959-2968.

von Knebel Doeberitz M (2001). New molecular tools for efficient screening of cervical cancer. Dis. Markers 17: 123-128. 